\title{
Circuito de producción y reproducción de los archivos de inteligencia: el Senvicio de Informaciones y el espionaje a las actividades culturales en el NE de Chubut (1969-1973)
}

\author{
Intelligence files production and reproduction circuit: The Information Service \\ and espionage of cultural activities in the NE of Chubut (1969-1973)
}

\section{Mónica Gatica \\ monicagracielagatica@yahoo.com.ar \\ Universidad Nacional de la Patagonia (UNP), Instituto \\ de Investigaciones Históricas (INSHIS), Argentina}

\author{
Axel Binder \\ axelbind@gmail.com \\ Universidad Nacional de la Patagonia (UNP), Instituto \\ de Investigaciones Históricas (INSHIS), Argentina
}

Recepción: 20 Abril 2021

Aprobación: 17 Mayo 2021

Publicación: 01 Junio 2021

Cita sugerida: Gatica, M. y Binder, A. (2021). Circuito de producción y reproducción de los archivos de inteligencia: el Servicio de Informaciones y el espionaje a las actividades culturales en el NE de Chubut (1969-1973). Aletheia, 11(22), e087. https://doi.org/10.24215/18533701e087
Resumen: En este artículo trabajaremos con algunos documentos de la carpeta "Legajo $\mathrm{N}^{\circ} 15$ Actividades Culturales" (APM, Chubut): un compilado del SI-Chubut sobre archivos de inteligencia con documentos procedentes de la SIDE, Policía de Chubut, la Armada Argentina y la Subsecretaría de Educación y Justicia de la provincia. La carpeta refleja el seguimiento a grupos de teatro y escritores de la región que, al menos desde 1960, fueron vigilados por su presunta "actividad comunista" y sospechados como potenciales "enemigos internos". Analizaremos cuatro casos en los que la actividad de inteligencia se centra en el ámbito educativo/ cultural: un pedido de antecedentes ideológicos sobre miembros de un grupo de teatro en 1969; otro pedido de información y antecedentes a raíz del Primer Encuentro Provincial de Escritores en 1970; la vigilancia a los participantes de la Temporada de Teatro de Verano de 1971 en Trelew; y el requerimiento SIDE sobre espectáculos de contenido ideológico marxista en febrero de 1973.

Palabras clave: Información, Vigilancia, Cultura, Policía, SIDE, Armada.

\begin{abstract}
In this article we will work with some documents from the folder "file No 15 Cultural Activities" (APM, Chubut): a compilation of SI-Chubut intelligence files with documents from the side, Chubut police, the Argentine Navy and the Undersecretariat of Education and Justice of the province. The folder reflects the follow-up to theater groups and writers in the region who, at least since 1960, were monitored for their alleged "communist activity" and suspected as potential "internal enemies". We will analyze four cases in which intelligence activity focuses on the educational/cultural field: a request for ideological background on members of a theatre group in 1969; another request for information and background following writers in 1970; surveillance of participants in the 1971 Summer Theatre Season in Trelew; and the SIDE requirement on performances of marxist ideological content in February 1973.
\end{abstract}

Keywords: Information, Surveillance, Culture, Police, SIDE, Navy. 


\section{INTRODUCCIÓN}

Este artículo se inscribe entre las producciones historiográficas abocadas al estudio de los procesos, modalidades y dispositivos represivos de la segunda mitad del siglo XX en Argentina (Aguila, 2018a; D’Antonio, 2018; Franco, 2016; Scatizza, 2017; Scocco, 2019; Bohoslavsky y Franco, 2020). Buscaremos contribuir al conocimiento sobre las configuraciones regionales, analizando algún material de archivos de inteligencia del Noreste (NE) de Chubut.

Cuando desde la archivística se habla del "contexto de producción" de los archivos, más que escudriñar razones a partir de la realidad histórica de la que emergen, se busca ante todo identificar la lógica de su procedencia y organización; esto es, la actividad que sus series documentales sedimentan, las razones de su preservación (o no) y sus posibilidades de acceso y consulta (Nazar, 2018). Nos gustaría agregar que el contexto de producción (al que en adelante nos referiremos como "circuito") refiere también a la dimensión material, técnica y social de su elaboración. ${ }^{1}$

La idea de abordar el "circuito de producción" de los archivos de inteligencia, nos posiciona no solo ante los mecanismos de acumulación de datos (recopilación y acopio), la división del trabajo en la confección del documento o las relaciones sociales que subyacieron a su producción; sino que también nos invita a considerar el espacio físico, los recursos e insumos, el personal (sus funciones y posiciones), los enlaces por los que circula la información, y los planes u operativos policiales/militares a la que sirven (consumo).

La propuesta conceptual consiste en asumir el documento de inteligencia, como un producto o mercancía, buscando reconstruir el know how técnico y las relaciones sociales que subyacen a la recolección de esa información y a la confección del documento. Pero atendiendo también a su posterior circulación, reproducción y, sobre todo, consumo; porque no hay que perder de vista que los materiales de inteligencia son el insumo clave para la instrumentación de planes represivos y para el control (vigilancia, seguimiento, persecución) de la población en general. ${ }^{2}$

Para comenzar a reconstruir la dinámica regional de la "inteligencia" y sus articulaciones, tomaremos como punto de partida el análisis de una serie documental producida por el Servicio de Informaciones Chubut (SI, delegación provincial de la SIDE). Se trata de una carpeta organizada por el SI-Chubut, que denominaron "Legajo n 15 de Actividades Culturales", donde se compiló parte de la información de inteligencia que resultó del seguimiento y vigilancia sobre escritores y grupos de teatro de la región, siempre bajo sospecha (como en casi todo el ámbito cultural, intelectual y educativo) de realizar "actividades comunistas" o de mantener vínculos con el "enemigo interno".

Esa carpeta de "Actividades Culturales" (1969-1973), forma parte del archivo represivo hallado que dio pie a la conformación del Archivo Provincial de la Memoria (APM) ${ }^{3}$; y si bien la SI-Chubut es el principal organismo productor, en el desarrollo de su actividad espía fue dejando huellas de contactos e intercambios con otros espacios de la "comunidad informativa". En ella, además de recortes periodísticos siguiendo la actividad teatral de la región (1970-1973), hay informes de la Policía de Chubut, memos del Servicio de Informaciones, comunicaciones de la SIDE, antecedentes ideológicos de artistas facilitados por la Armada y la Policía Federal y hasta informes de la Subsecretaría de Educación y Justicia de la provincia. Toda información clasificada con carácter reservado, secreto y urgente. 


\section{LA ESTRATEgia REPRESIVA POST-CoRdobazo Y LA CENTRALIDAD DE LOS ÓRganos DE INTELIGENCIA}

Las doctrinas militares que centralizaron la necesidad de seguridad y orden público justificando la intervención política de las Fuerzas Armadas, se consolidaron a finales de los años 50 y 60 en Francia y USA a partir de los conflictos de liberación nacional en Argelia, Cuba y Vietnam. La Doctrina de Seguridad Nacional (DSN) con sus elementos de contrainsurgencia y sus consideraciones geopolíticas en el marco de la "Guerra Fría”, constituyó la plataforma estratégica que habilita, desarrolla y da contenido específico (e histórico, combinando "seguridad y desarrollo") a estas prácticas orgánicas de inteligencia. Por su parte, la doctrina francesa de la guerra revolucionaria (DGR), aportó los fundamentos tácticos y técnicos para la represión clandestina de un "enemigo interno" al que se presumía infiltrado entre la propia población. Razón por la cual la "inteligencia" ocupa un lugar central en esa metodología clandestina de represión contrainsurgente. ${ }^{4}$

En Argentina, ambas vertientes doctrinarias (DSN y DGR), fueron habilitando un proceso en el que progresivamente se identificó a todo aquel que era activista político, simpatizante de izquierda o bien disidente de todo género, con el "enemigo interno" que se debía erradicar. Los manuales militares de la época afirmaban que "una inteligencia precisa, detallada, oportuna y permanente, concretada en el análisis de las características de la zona urbana y de la situación del enemigo, será esencial para el éxito de las operaciones contra la subversión urbana". ${ }^{5}$ La creación de la Central Nacional de Inteligencia (CNI) durante la "Revolución Argentina" (1966), prevista para suministrar información estratégica al Consejo Nacional de Seguridad (CONASE), es elocuente de cómo la "comunidad informativa" fue un engranaje esencial del aparato represivo de las dictaduras de "Seguridad Nacional".

Tras las revueltas obreras de 1969, especialmente luego del Cordobazo, la estrategia de las FF.AA. se orientó a reprimir a un "enemigo interno" que además de ser comunista/izquierdista, pasa ahora a ser considerado también como subversivo. De manera explícita, la "Directiva Fijando Políticas y Estrategias para Eliminar la Subversión” (COS-13) ${ }^{6}$ del año 1969-1970, en su distribución de responsabilidades, fijaba para la CNI la tarea de dirigir:

el centro de gravedad de las actividades de Inteligencia para posibilitar la destrucción de la estructura subversiva y neutralizar los estallidos de violencia, teniendo en cuenta la exigencia de emitir informes en oportunidad a fin de prevenir las acciones subversivas y permitir el mantenimiento de la iniciativa por parte de las fuerzas legales, para lo cual asignará definidas exigencias y responsabilidades a los organismos de inteligencia cooperantes (de las FF.AA y de las fuerzas de seguridad).

La Directiva COS-13, impartía precisiones operativas para la "lucha antisubversiva" que la ley de Seguridad Nacional 16.970 no conseguía dejar suficientemente claro -sobre todo en lo que respecta a objetivos, atribuciones y responsabilidades-. Las generalidades sobre la defensa nacional y el enemigo interno (claramente subsidiarias de la Doctrina de Seguridad Nacional), ya no se ajustaban a las pretensiones represivas del régimen "post-cordobazo". Las modificaciones en la estrategia represiva rápidamente encuadraron la conflictividad social en el paradigma militar de lucha contrainsurgente, que para mediados de 1971, asumía oficialmente la "Hipótesis de guerra en el marco interno", subordinando a las policías y colocando a las Fuerzas Armadas al frente de la "lucha contra la subversión".

COS-13 (como en otras directivas posteriores) preveía además que un organismo del Estado (en este caso la CNI) ejerciera el manejo centralizado de la información y de las actividades de inteligencia (de la SIDE, las policías provinciales y fuerzas armadas) para servirle al CONASE en sus planes. En la asignación de nuevas funciones y responsabilidades que emanaban de la directiva presidencial COS-13, se fijaba que la CNI proporcionaría:

por medio de un informe periódico mensual y/o cuando la situación así lo imponga, inteligencia estratégica centralizada y evaluada", manteniendo además 'enlace técnico funcional con los organismos afines que integran la llamada Comunidad 
Informativa' debiendo ejercer además el control sobre toda clase de prensa oral y escrita, cine, radio y televisión, a los fines de impedir, de acuerdo a la legislación vigente, que se distorsione ideológicamente la realidad nacional. ${ }^{9}$

En ese marco represivo general, de transición y con renovados objetivos militares e imperativos de inteligencia (que no hacen sino perfeccionar y profundizar viejas prácticas de control social y político), se inscribe este trabajo. A partir del análisis de la sección "Legajo N¹5. Actividades Culturales" del archivo del SI-Chubut, buscaremos reconstruir parte de las lógicas y modalidades de la dinámica represiva regional; en especial su circuito de inteligencia (a quienes se espiaba, quienes lo hacían, cuándo, cómo y para qué). Esto hará que nos apartaremos de la instancia represiva de la persecución y castigo (detenciones, allanamientos, censuras, secuestros, torturas y masacres), para centrarnos en otro tramo del trayecto represivo: el de la vigilancia y control de quienes se presumían como "enemigos internos" (en este caso, en el ámbito cultural). Más allá de las situaciones puntuales en las que la dictadura revela abiertamente su carácter autoritario criminal (siendo la Masacre de Trelew -de agosto de 1972- la más paradigmática de ellas), el sistema represivo en la región pareciera haberse abocado principalmente a controlar, espiar y vigilar a la población. ${ }^{10}$ Para comenzar el armado de ese rompecabezas represivo y su trama de inteligencia, analizaremos cuatro casos que consisten en pedidos de informes de inteligencia del ámbito educativo/cultural: uno de antecedentes ideológicos sobre miembros de un grupo de teatro en 1969; un pedido de información y antecedentes a raíz de un encuentro provincial de escritores en 1970; el seguimiento a la Temporada de Teatro de Verano en 1971; y finalmente un requerimiento de información sobre espectáculos teatrales con "contenido ideológico marxista”, en febrero 1973.

\section{1) Febrero de 1969: Pedido de antecedentes ideológicos}

La primera sección de documentos que integran la serie de "Actividades Culturales" del SI-Chubut, revela la vigilancia ideológica a la población local en el seguimiento al grupo Teatro Estudio Trelew (TET). Se inicia con un pedido de la Policía de Chubut que promueve el intercambio de mensajes e información con otras dos agencias: la Base Aeronaval Alte. Zar (BAAZ) y la Policía Federal.

Las 9 fojas que registran la actividad de ese requerimiento se desprenden del prontuario policial de Rodolfo Miele, que se compone de 60 fojas totales. ${ }^{11}$ Es decir que -originalmente- la información recabada que suministran la Armada y la Policía Federal fue a engrosar su legajo policial. Presumimos que fue el propio SIChubut el que separó esas fojas específicas referidas al teatro, para incorporarlas a la carpeta de "Actividades Culturales"; y duplicando así la información, que deja de estar personalizada/focalizada (prontuario policial de Miele) para ser manejada como información estratégica de carácter colectivo, sobre un grupo o actividad determinada de la población.

La documentación da cuenta de una solicitud al jefe de la Base Aeronaval de Trelew, en la que se le requiere información sobre grupos de teatro y demás "antecedentes que pudieran registrar, en especial ideológicos de las personas que se mencionan a continuación”. ${ }^{12}$ La Armada enviaría datos sobre 5 personas que tenían registradas en su "Sección de Información y Seguridad”, información que sería reproducida al ser incorporada -como dijimos- a los legajos policiales, ampliando así su base de datos sobre personas: "se tomó nota en los respectivos legajos", dice en rojo y en cursivas escritas a mano, en el documento de respuesta de la Armada.

A propósito del mismo, nos gustaría detenernos en dos cuestiones: una es el sello en la parte inferior de la hoja, en rojo y mayúsculas advierte: "ESTOS ANTECEDENTES SON ELEMENTOS DE ORIENTACIÓN Y NO DE PRUEBA, SU DIFUSIÓN ESTÁ PENADA POR EL DECRETO LEY ART. 634 - 636- 772 y 780 del C.J.M". ${ }^{13}$ El aviso en sí (dando cuenta de una ambigua zona gris entre lo legal y lo clandestino) delata la "naturaleza" real del uso de la inteligencia: la persecución política. Fernández Picolo (2014) explica que la detención de Elvio Ángel Bel en 1970 (docente y militante del Partido Comunista), se sostuvo sin mayor respaldo probatorio que el de la presunción de infracción a 
la ley 17.401, dados sus antecedentes ideológicos. Los mismos, se basaban en un informe de Prefectura que lo conceptuaba como "ACTIVISTA COMUNISTA". ${ }^{14}$ Observa además que el informe, contiene documentación agregada de la SIDE, en la que recomendaba la 'EXCLUSIÓN PARA OCUPAR CARGOS EN LA ADMINISTRACIÓN PÚBLICA' de Elvio Ángel Bel "de acuerdo a un informe producido por la Comisión Asesora para la Calificación de Ideología Extremista (CACIE) con fecha 30 de octubre de 1968" (Fernández Picolo, 2014, p. 125). De momento, la imposibilidad de disponer de otros prontuarios y archivos policiales de inteligencia no nos permite cotejar tratamientos similares en otros.

La otra cuestión que nos interesa destacar del documento de respuesta de la Armada es la aparente "especialización" que asume la Base Zar en materia de espionaje político-ideológico. Los fichajes sobre las personas que remite la Base Zar se componen de una información "mixta" de origen policial y propio. Por ejemplo, en los antecedentes que la Armada remite sobre Miele, se aprecian claramente 2 vertientes informativas: una primera -al comienzo- donde se suministran los datos filiatorios (domicilios, estado civil, radicaciones, trabajos, etc.), contravenciones y causas judiciales; información que originariamente produce y provee un prontuario policial. Seguidamente se aprecia la segunda vertiente, que incorpora datos de naturaleza política-ideológica, diferente a los que "típicamente" manejaba la policía. Esto nos permite afirmar dos cuestiones: que la Armada recopila y produce información de inteligencia sobre los habitantes de la región; y que la Policía se encuentra "actualizando" sus propios registros reuniendo y cruzando datos que obtiene de la comunidad informativa.

El pedido que cursa la Policía de Chubut a Coordinación de la Policía Federal estuvo más enfocado, centrándose sólo en las 6 personas (de la lista de 18 que habían enviado a la BAAZ) de las cuales tenían datos filiatorios a partir del registro prontuarial. Sumaron en el requerimiento a Coordinación Federal datos sobre José Macías (otro militante comunista), que la policía no había solicitado a la Armada. La Policía Federal remite un largo informe de 3 hojas, 2 de las cuales están dedicadas a Macías. Esa información se componía a su vez de 2 registros: por un lado, el "propio" de la Federal que aportaba información ambiental suya de los años 1966, 1967 y $1968^{15}$; por otro, las entradas que sobre el “informado" también tenía otro "organismo afín” (del que se sirve la Policía Federal), sin explicitar la institución productora.

Los datos sobre R. Miele que facilita la Policía Federal (cuya información "ambiental” ya lo posicionaba

como "Comunista") reseñan cosas diferentes de las que apunta el informe de la Armada. ${ }^{16}$ Es de notar que la información "ideológica" sobre Miele que maneja la Armada, es entre los años 1959 y 1963; mientras que la información "ambiental” que suministra la Policía Federal es entre 1965-1966. En líneas generales, comparando la "calidad ambiental" de la información que propician ambas agencias, se advierte que la información que maneja la Policía Federal es mucho más densa, completa y narrada que la suministrada por la Sección "Información y Seguridad" de la BAAZ (que constituye más bien un punteo de datos relativamente dispersos). A diferencia de la Armada, se nota que los datos de filiación que maneja Coordinación Federal son muchos más escuetos (lo que sugiere un vínculo informativo -enlace- más estrecho entre la Policía de Chubut y la BAAZ).

Todo ello nos sugiere la presencia de más de un organismo recabando información in-situ y produciendo archivos de inteligencia. Nos preguntamos ¿qué otra dependencia, además de la Armada y la Policía Federal, se encontraba produciendo y recopilando información política-ideológica? ¿Cuál sería ese otro "organismo afín" que también realizaba ese tipo de inteligencia en la región? ¿y desde cuando lo hacía?, son preguntas que se desprenden de este caso, y que guían nuestras investigaciones en curso.

\section{2) Junio de 1970: ENCUENTRo DE ESCRITORES PATAGónicos}

El 11 de junio de 1970, se realizó en la Biblioteca Popular "Agustín Álvarez" de Trelew, el "Primer Encuentro de Escritores de la Patagonia”, ocasión en la que se anunciaría la conformación de una filial de la 
Sociedad Argentina de Escritores (SADE). ${ }^{17} \mathrm{El}$ evento que congregaría unos 30 participantes de las provincias de Santa Cruz, Chubut, Río Negro, Neuquén, La Pampa y Buenos Aires, dio lugar a un requerimiento de información de parte del SI-Chubut, solicitando antecedentes de las personas "a fin de detectar las posibles infiltraciones en la entidad". ${ }^{18}$

El pedido estuvo dirigido en principio a la SIDE y a la Jefatura de Policía de Chubut; y cuando estos últimos notifican haber realizado el mes anterior un informe de similares características para la Subsecretaría de Educación y Justicia de la provincia, el SI-Chubut cursa también un pedido de antecedentes al ministro de la cartera, solicitándole la nómina de participantes, la representación que investían, sus domicilios y los documentos de identidad declarados ${ }^{19}$. En conjunto, el intercambio de información que articula al SI-Chubut (iniciador), SIDE, la Jefatura de Policía Provincial y la Subsecretaría de Educación y Justicia de Chubut, obra de 18 documentos (fojas) producidos entre junio y diciembre de 1970. El informe de inteligencia en el que se notifican los antecedentes político-ideológicos de 11 personas ${ }^{20}$, viene a dar cuenta de otros circuitos de producción y circulación de información. Todo indica que la mayor parte de la información fue recolectada y suministrada por la Policía de Chubut.

El único documento de respuesta de la SIDE en esta sección (que lleva un sello de CACIE) ${ }^{21}$, fue solicitando rectificación de datos sobre una de las personas mencionadas. Sin embargo, su involucramiento en el pedido permite dilucidar uno de los posibles circuitos de inteligencia: al contar el evento con escritores de otras provincias, el SI-Chubut pide explícitamente al Departamento de Informaciones y Fichajes de la SIDE, “información que podrán aportar los señores Delegados de SI NEUQUÉN, LA PAMPA, RÍO NEGRO Y SANTA CRUZ, en base a la actuación pública de los mismos en sus respectivos lugares de radicación". ${ }^{22}$ El requerimiento refleja la posible triangulación entre agencias para obtener información sobre otras jurisdicciones provinciales, actuando la SIDE como pivote.

Lo mismo permite inferir la información que suministra la Jefatura de Policía de Chubut sobre las personas de otras provincias, utilizando a la Policía Federal como articulador en la mediación. El informe explicita que sobre uno de los escritores de La Pampa se "esperan antecedentes de la Policía Federal" ${ }^{23}$ Esto nos hace pensar que los datos del informe pudieron haber sido recabados por la policía provincial, más no producidos. Que antes bien, fueron otros miembros de la comunidad informativa los productores originales de esa información especializada.

A propósito de ella, la ficha de Luis Feldman Josín (director del Diario Jornada) revela información acumulada desde 1939 hasta 1966, concentrándose a partir de 1960 en aspectos de índole mayormente ideológica (básicamente su relación con el "comunismo"): como que el 25 de octubre de 1960 "publica un artículo titulado "la literatura comunista"; o que "luego de su regreso de Alemania, país al que viajó por invitación del gobierno alemán, publicó una serie de artículos de contenido netamente anticomunista, comentando la vida de opresión que sufre el pueblo alemán detrás de las alambradas de Berlín"; o que en 1962 poseía un "negocio de librería en Esquel, donde la mayoría de los libros que allí se venden son de tendencia izquierdista" ${ }^{24}$. Al final de su informe, se agregó una suerte de balance o evaluación de Feldman Josín, que concluye -entre otras cosas- que:

Los antecedentes del causante no permiten abrir un juicio definido sobre su ideología, dado que de su accionar dentro del comunismo lo único concreto y confirmado por el mismo, es lo relacionado con la distribución del Diario 'La Hora'. A esto se contrapone la serie de artículos anticomunistas publicados en el diario 'Jornada', luego de su viaje a Berlín (...) De todo lo expuesto, se deduce que se trata de un individuo sumamente hábil, que hasta el presente se ha adaptado a las cambiantes situaciones del país, lo que lo convierten en una persona de cuidado. ${ }^{25}$

El grado de detalle de la información, la naturaleza política de la misma y las apreciaciones valorativas como la precedente, nos sitúan otra vez ante la pregunta por el organismo productor: ¿es información generada por la propia institución policial? ¿la produce la Base Zar? ¿el SI-Chubut? ¿o cada cual manejaba y construía su propio archivo? ¿o alguno hegemonizaba la vigilancia ideológica? Si bien sabemos que parte de la información 
es suministrada por la Policía Federal, seguimos sin lograr precisar quién es el productor de base (quien aporta la mano de obra "espía" o quien recoge la información en el terreno/campo).

Identificar con certeza el organismo productor del informe, o discernir cuál es el tipo de información específica que pudo haber suministrado cada institución, es una de las dificultades que presenta este caso. Todo parece indicar -tal como señala el APM- que el informe fue elaborado por la Jefatura de la Policía provincial, lo que marcaría una notable diferencia con el caso anterior de 1969: en ese entonces el organismo era el que solicitaba antecedentes políticos/ideológicos; mientras que en 1970, parece estar en condiciones de disponer y suministrar ese tipo de datos, ante el requerimiento de terceros (SI-Chubut, en este caso). ${ }^{26}$ Pareciera que la Policía de Chubut retoma el anterior trabajo de inteligencia realizado para la Sub-Secretaría de Educación y Justicia, ajustándolo al posterior requerimiento del SI.

De lo que no hay dudas es que la policía provincial fue aprovechando este tipo de circunstancias y requerimientos (encuentro de escritores, por ej.) para mediar con otras agencias e ir recopilando y acumulando información de tipo "ambiental" o política-ideológica, que un año antes parecía no manejar o disponer. Pero tal vez lo más trascendente del caso del "Encuentro de escritores" de 1970, es que la Subsecretaría de Educación se haya adelantado un mes antes al requerimiento de antecedentes al SI-Chubut; esto viene a dar cuenta que la cartera provincial no sólo proporcionaba información (como veremos en siguiente caso), sino que también la requería. Este antecedente de colaboración (enlace) demuestra que la tarea represiva y su trama de inteligencia no se circunscribieron a las fuerzas de seguridad, sino que involucró y articuló otras reparticiones del estado.

\section{3) Enero-febrero de 1971: Temporada de Teatro de Verano (1971)}

Este caso de seguimiento a la actividad cultural gira en torno al encuentro que organizó el Teatro Estudio Trelew, invitando a grupos vanguardistas de Buenos Aires a realizar talleres y muestras en la región. También participaron directores como Roberto Espina, Edgar González y Renzo Casalli que realizaron exposiciones y dictaron cursos. Las actividades, que incluyeron 6 obras teatrales, se desarrollaron entre el 16 de enero y el 21 de febrero.

El primer documento de la sección (de unas 14 fojas), lo produce la Subsecretaría de Educación y Justicia en enero de 1971, presumimos que por requerimiento del SI Chubut, a quien está dirigido. En esa contestación se eleva la nómina de elencos y participantes de la Temporada de Teatro de Verano: las obras a presentar, sus intérpretes, vestuaristas, maquilladores, dirección, iluminación; o los cursos a dictarse y las fechas, tanto de presentaciones como de talleres (información pública también suministrada por los medios de comunicación). Al mes siguiente, sobre el cierre de la "temporada de Teatro", SI-Chubut volvería a pedir a la Subsecretaría de Educación y Justicia, esta vez, una evaluación sobre la "calidad de los conjuntos actuantes, calidad y fondo de las obras representadas, alojamiento de los elencos y directores, colaboración moral y material de la Provincia, repercusión en el medio ambiente y conclusiones y experiencia recogida por el ente específico Provincial". ${ }^{27}$

El resultado del "informe" que genera la Subsecretaría, que reviste carácter confidencial, no aporta mayores elementos de inteligencia sobre la cuestión ideológica/política o que sirviesen para encuadrar la actividad como "comunista" o "subversiva". Hasta la propia publicación del evento que realiza la revista TEATRO/70, sin ser secreta ni clandestina, brinda más información sobre el perfil ideológico-político del encuentro, y las discusiones que se debatían sobre el lugar del arte y la función política del teatro (discusión que dividía a los dos grupos de teatro locales: el Grillo y al TET). ${ }^{28}$

El informe realiza una evaluación general, abordando la calidad de los conjuntos, el fondo de las obras, la colaboración material de la provincia y su repercusión local. ${ }^{29}$ En suma, antes que un informe típico de inteligencia constituye más bien una crítica o reseña de las obras presentadas: 
El 'Teatro de los Jóvenes' de la Municipalidad de Merlo, es un discreto conjunto teatral, donde se destacó la presencia del primer actor, y, en cierta medida, la primera actriz. Los restantes integrantes no están a la altura de una nuestra que pretende demostrar las bondades del teatro actual. Nivel desparejo donde la dirección no alcanza a disimular defectos de la puesta en escena. ${ }^{30}$

No obstante, no dejan de estar "atentos" a la cuestión ideológica que se sobreentiende le interesa al SIChubut (receptor): por ejemplo se consignó que las "Improvisaciones Premeditadas" de Roberto Espina fue un "espectáculo ameno que insertó algunas críticas sociales"; o bien que la obra "El Sótano" del Grupo Joven de la localidad de Merlo “...presentó una problemática de tipo social que dio lugar a diversas interpretaciones y deja librado al juicio del espectador las soluciones a los problemas planteados, si bien se nota en los autores un deseo de cambio de las actuales estructuras sociales". ${ }^{31}$

Este caso, en particular, nos abre a la perspectiva de pensar que estos documentos son también algo más que un archivo de represión (por más que, como dijimos, su finalidad haya sido servir a ella). El caso de la Temporada de Teatro 1971, agrupa archivos de inteligencia del SI-Chubut, generados por la Subsecretaría de Educación, en la intención de reprimir, perseguir o controlar política e ideológicamente a las personas. Su eficacia, sin embargo, resulta pobre en términos de "inteligencia"; de hecho, en ningún momento llega a darse cuenta de que fue R. Miele (miembro del grupo de teatro TET, objeto de espionaje en el primer caso que presentamos) el que organizó el encuentro teatral. Información que, dicho sea de paso, tampoco se encuentra consignada en su prontuario policial. En su lugar, el requerimiento del SI-Chubut parece acabar produciendo una suerte de crítica literaria, que además de preservar los datos "técnicos" de las obras, constituye una devolución de un atento espectador, que más que mirar con ojos de "espía" interpretó las obras con carácter literario y teatral.

\section{4) FEBRERo 1973: INFORMACIÓN SOBRE ESPECTÁCUlOS DE CONTENIDO IDEOLÓGICO MARXISTA}

Este último caso, que cronológicamente cerraría la serie de "Actividades Culturales" del SI-Chubut, se inicia con un pedido de la SIDE solicitando información permanente "...sobre la actividad de Grupos Teatrales y/o espectáculos en general, que difundan mensajes de contenido ideológico marxista”. ${ }^{32}$ Siguiendo la cadena de requerimientos (y trazando otro de los circuitos de inteligencia), el SI-Chubut traslada el pedido a Policía Provincial (al Dpto. de Informaciones Policiales-DIP) para "remita la información relacionada con la difusión que se realiza en la provincia de cualquier tipo de acción psicológica en favor de la ideología comunista-marxista" o "cualquier tipo de espectáculo en general, público o privado, que difunda mensajes del mismo contenido extremista". ${ }^{33}$

En respuesta la Jefatura de Policía (con sello del DIP), remitiría al delegado provincial del SI tres informes sobre los grupos de teatro "Genpin", "El Grillo" y "Nuevo Grupo". Lo curioso, es que un año antes, era el propio DIP que solicitaba a través de su Jefatura información al SI- Chubut sobre el elenco "El Grillo" ${ }^{34}$ De lo que ya no queda duda es que, hacia fines de la dictadura, la policía provincial ya desarrolla actividades de vigilancia ideológica, manejando y compartiendo información propia con otras agencias, algo que aparentemente no estaba en condiciones de hacer hacia 1969 (a juzgar por el primer caso analizado). El volumen informativo en los partes sobre los tres grupos de teatro demuestra que para 1973 la Policía de Chubut parece tener a disposición un caudal importante de información personal y de ambiente de los distintos elencos y artistas (y con seguridad también de militantes políticos, organizaciones sociales y sindicatos; pero hasta que podamos dar con esas otras fuentes será sólo una hipótesis de trabajo).

Por ejemplo, sobre la obra "Veni que hay amor y bronca" presentada por el grupo "Genpin" (29/11/1972) el parte policial, además de tomar nota de la cantidad de asistentes y algunos de sus nombres, dice: 
informe producido por este departamento con motivo de la obra: la misma se desarrolla en dos actos (...) el argumento de la obra versó en los problemas existentes en el ámbito nacional, dejando ver un neto corte izquierdista insitando (sic) a la juventud que realiza estudios en facultades e institutos de enseñanzas que se vuelquen a la lucha por conseguir una patria libre y socialista. Deja ver la desigualdad existente en nuestro país y las prohibiciones de que era objeto la población por parte del 'Gorila Gobierno'. 35

El SI-Chubut elevó esos informes policiales a la Inspección Delegaciones Regionales y Enlaces Provinciales de la SIDE. Y más tarde ese mismo día, también informó a la SIDE que el grupo de teatro "El Grillo" tenía intenciones de "presentar para el 22 de agosto próximo una farsa o sátira de sucesos acaecidos Base Aeronaval Trelew con motivo cumplirse primer aniversario muerte 16 guerrilleros evadidos cárcel de Rawson. Se ampliará información. Valoración C.3" ${ }^{36}$ Esta es la última documental obrante en la carpeta previa al recambio institucional de la salida democrática.

Otro parte de la SIDE que no integra la carpeta de actividades culturales, pero forma parte del acervo documental del APM Chubut, arroja luz -e inquietud- sobre la "suerte" de la información de inteligencia recabada durante la dictadura; archivo que no pensaba ser legado a la administración democrática del estado, lo que es un aporte central para pensar las continuidades/rupturas de las agencias represivas y sus tramas de inteligencia.

El parte fue elaborado por la SIDE el 5/04/1973 y dirigido al delegado provincial del SI-Chubut, adjuntándole las "DIRECTIVAS PARA EL TRATO DE DOCUMENTACIÓN” donde se explica el procedimiento a seguir con "determinada documentación existente de índole variada (política, subversiva, antecedentes solicitados y/o evacuados, etc.)". ${ }^{37}$ Se recomendaba relevar la documentación existente "especialmente años 1972 y 1973" y clasificarlas por factores y por su contenido: "De las calificadas como muy trascendentes y trascendentes, separar aquellas que aún tengan valor para el trabajo informativo subversivo ideológico (marxismo, maoísmo, troskysmo, etc.)”. Seguidamente se debería remitir un inventario de la información consignando volumen, para posteriormente enviar la documentación por Valija Postal; "con el resto de la documentación que no sea remitida, aunque no posea valor por su contenido subversivo ideológico, se la podrá incinerar, pero labrando un acta" en el que debería especificarse "que se procede a incinerar por haber perdido actualidad y valor informativo.” El documento finaliza con la siguiente apreciación:

En síntesis, el momento político nacional y provincial prácticamente ha terminado a la luz del resultado electoral. Pero el accionar subversivo, atentatorio contra un sistema de vida argentino, regido por preceptos Constitucionales, continúa y probablemente continuará, aún con las autoridades elegidas. Prueba de ello es que entre el 11 de marzo y la fecha siguen sucediéndose, con igual intensidad, hechos insurreccionales (robos, secuestros, atentados, intimidaciones, asaltos, etc.). Tales hechos no tienen sino un fondo ideológico y su acción no repara en figuras o matices políticos, sin distinción. Por último, se estima conveniente que la tarea se inicie a la brevedad y se de por terminada el 02 MAY $73 .{ }^{38}$

\section{CONSIDERACIONES PRELIMINARES}

La vigilancia y el seguimiento a la actividad cultural revelan parte del circuito de producción y reproducción (circulación) de la información de inteligencia en el NE de Chubut. El análisis de los documentos que compila el SI-Chubut, y cuyos principales productores son la Armada, la Policía Federal y la Policía de Chubut, nos permiten comenzar a reconstruir los lazos y el funcionamiento de la "comunidad informativa".

Hemos podido observar en esa documental del SI-Chubut sobre la actividad cultural, un desplazamiento en la categoría del "enemigo interno" que manejan las fuerzas armadas y de seguridad, advirtiendo que hasta fines de la década del "60 son definidos como "comunistas" y/o "izquierdistas"; y hacia 1972/73 ya son catalogados como "marxistas" y "subversivos" ${ }^{39}$ La impresión general que nos dejan esos documentos producidos en la coyuntura histórica "post-cordobazo" (1969-1973), es la de un periodo de profesionalización donde las distintas agencias de inteligencia se encuentran aggiornando sus prácticas y 
requerimientos al nuevo paradigma represivo, de corte contrainsurgente y orientado a la lucha interna contra la "subversión". 40

Puestos en perspectiva, esos documentos de la carpeta de "Actividades Culturales" (y los requerimientos de información que contienen) revelan un creciente protagonismo del SI-Chubut y de la Policía provincial. Entre 1970 y 1973 puede apreciarse cómo el SI (que pareciera funcionar como la delegación provincial de la SIDE), comienza a hacerse un lugar dentro de la comunidad informativa, primero acumulando información de otras agencias y luego produciendo material de inteligencia propio, a partir de los requerimientos que le realiza la sede central.

Situación similar parece ser la de la Policía del Chubut, que comienza la sección de documentos solicitando información de tipo ideológica/política en 1969; en 1970 ya se encuentra suministrando información ante requerimientos de terceros (Subsecretaría de Educación y SI-Chubut) y para 1972 ya se tienen registros de agentes propios desarrollando tareas de vigilancia, a cargo del Departamento de Informaciones Policiales (DIP-D2), especializándose en el espionaje político y control ideológico.

Esto no significa que la Policía de Chubut no hubiese realizado desde sus inicios tareas de vigilancia política y de control social; hemos observado en algunos prontuarios policiales de las décadas del ' 40 y ' 50 sellos de la "División de Informaciones". Lo que queremos señalar es que desde la década del "70 se inicia una especialización bajo un paradigma diferente que presenta una variante en la concepción del enemigo interno y en su forma de "combatirlo" (del "comunista" a al "insurgente/subversivo"), lo que fue dando lugar a una profesionalización que presumimos comienza en Chubut entre 1971-72, con la creación del Departamento de Informaciones Policiales (DIP). ${ }^{41}$

Atendiendo a la participación de la policía en estos documentos que compila el SI-Chubut, puede advertirse que el rol de mediación que ocupa dentro de la comunidad informativa (solicitando información y cumpliendo requerimientos) le permitió "stockearse" en esta primera etapa de "profesionalización", de información que fue engrosando su archivo propio de inteligencia. Para 1972, ya tenemos registros de que la policía (DIP) produce su propio material, tal como venían haciendo la Base Zar y la delegación de la Policía Federal en Rawson. Y aunque de momento no podamos estipular una jerarquización ni una diferenciación respecto de la calidad y contenido de la información que genera y recopila cada uno, todo parece indicar que la Armada desde 1971 pasa a centralizar la responsabilidad operativa y a dirigir la comunidad informativa. ${ }^{42}$

Esas hipótesis, y en especial la reconstrucción del derrotero de la institución policial, su organigrama, reglamentación y atribuciones dentro de la "comunidad informativa", conforman las líneas de investigación que venimos desplegando desde el Instituto de Investigaciones Históricas (INSHIS), en torno al Archivo de Prontuarios Policiales de Chubut (Pérez Álvarez y Gatica, 2019; Tapia, Chávez y Vezub, 2020; Binder, 2020).

Consideramos que los prontuarios policiales son una punta de lanza para comenzar a reconstruir la trama represiva y de persecución política en la región. No solo porque la información de base (datos de filiación e identificación) es producida por la institución, sino porque el prontuario en sí representa un "mapa" que va dejando registro de los distintos intercambios entre agencias y departamentos (lo que ingresa, sale, se pide o expide). Hemos visto como el prontuario de Rodolfo Miele registra ese flujo de intercambios informativo consignado sus productores, lo que nos permite advertir los vínculos, especializaciones y jerarquías de la comunidad informativa.

La dificultad con la que nos encontramos (además de la destrucción de documentos), es la gran dispersión y fragmentación del archivo policial. De momento, no hemos podido ubicar en el Archivo de Prontuarios Policiales (en guarda en el INSHIS) las secciones de "orden social o político", u otros prontuarios con información específica de inteligencia. Algunos de estos últimos documentos, fueron separados y se encuentran alojados en el APM; otros se encuentran en el Museo Policial, con ciertas dificultades de conservación y acceso para su consulta. 
El haber podido acceder a esta pequeña serie "cultural" del SI-Chubut, nos permitió comenzar a desandar el arduo camino de reconstrucción de las redes represivas y de inteligencia en la región (y sus vínculos del orden nacional), demostrando la potencialidad de estos archivos. Pero sobre todo revela la necesidad de trabajo en equipo, por lo que apostamos a articular esfuerzos entre la investigación jurídica e histórica, la preservación documental y el rescate de la memoria; ese el rumbo de trabajo que desde el INSHIS, la Subsecretaría de Derechos Humanos, la Jefatura Policial y la Municipalidad de Trelew hemos venido trazando.

\section{REFERENCIAS}

Aguila, G. (2013a). La represión en la historia reciente argentina: fases, dispositivos y dinámicas regionales. En G. Aguila y L. Alonso (Coords.). Procesos represivos y actitudes sociales: entre la España franquista y las dictaduras del Cono Sur (pp. 97-121). Buenos Aires: Prometeo Libros

Aguila, G. (2013b). Las tramas represivas: Continuidades y discontinuidades en un estudio de caso. La Dirección General de Informaciones de la Provincia de Santa Fe, 1966-1991. Sociohistórica (31). Recuperado de: http://w ww.memoria.fahce.unlp.edu.ar/art_revistas/pr.5742/pr.5742.pdf

Aguila, G. (2018a). La represión en la historia reciente como objeto de estudio: problemas, novedades y derivas historiográficas. En G. Aguila, G. et al., La historia reciente en Argentina. Balances de una historiografía pionera en América Latina (pp. 55-72). Buenos Aires: Imago Mundi.

Aguila, G. (2018b). Policías, represión y 'lucha antisubversiva': exploraciones sobre el rol de las policías en el accionar represivo de los años 70 en Argentina. Folia Histórica del Nordeste, (32), 121-146.

Binder, A. (2020). El archivo de prontuarios policiales de Chubut: hacia una descripción y valoración de los documentos. Revista Electrónica de Fuentes y Archivos, 11(11), 215-240.

Bohoslavsky, E. y Franco, M. (2020). Elementos para una historia de las violencias estatales en la Argentina en el siglo XX. Boletín Del Instituto De Historia Argentina Y Americana Dr. Emilio Ravignani, (53). Recuperado de: http s://doi.org/10.34096/bol.rav.n53.8018

D'Antonio, D. (2018). (comp.). Violencia, espionaje y represión estatal. Seis estudios de caso sobre el pasado reciente argentino. Buenos Aires: Imago Mundi

Fernández Picolo, M. (2014). Los Presos Políticos y la Solidaridad. En AA.VV. Trelew, esa masacre que aún es fuego (pp. 107-147). Rawson: Secretaría de Cultura de la Provincia del Chubut.

Franco, M. (2016). La represión estatal en la historia argentina reciente: problemas, hipótesis y algunas respuestas tentativas. En G. Águila, S. Garaño y P. Scatizza (eds.), Violencias de Estado. Formas y dinámicas represivas en la historia reciente argentina: nuevos abordajes a 40 años del Golpe de Estado (pp. 15-43). Buenos Aires: UNLP.

Funes, P. (2007). Los libros y la noche. Censura, cultura y represión en Argentina a través de los Servicios de Inteligencia del Estado. Dimensões, (19), 133-155.

Funes, P. (2008). Desarchivar lo archivado. Hermenéutica y censura sobre las ciencias sociales latinoamericanas. Iconos Revista de Ciencias Sociales, (30), 27-39. Recuperado de: https://doi.org/10.17141/iconos.30.2008.246

Gatica, M. (2010). Del abordaje teórico al análisis empírico: un archivo de la represión en Rawson, Chubut. Anuario de la Escuela de Historia, (22), 156-182.

Margaria, P. y Schnyder M. C. (2013). De la represión al control disciplinante: reformulaciones y continuidades del DIP de la policía santiagueña en el pasado reciente. Ponencia presentada en las VII Jornadas Santiago Wallace de Investigación en Antropología Social. Sección de Antropología Social. Instituto de Ciencias Antropológicas. Facultad de Filosofía y Letras, UBA, Buenos Aires.

Nazar, M. (2018). Secretos, reservados y confidenciales: la producción de la información de las fuerzas armadas y de seguridad como fuente para la historiografía). Estudios Sociales del Estado, 4(7), 243-264. Recuperado de: http s://doi.org/10.35305/ese.v4i7.15 
Oliveira-Cézar, M. (2005). Del modelo contrarrevolucionario francés al modelo aplicado en la Argentina del Proceso. América: Cabiers du CRICCAL, (33), 121-129. Recuperado de: https://www.persee.fr/doc/ameri_0982-9237 _2005_num_33_1_1713

Pérez Álvarez, G., y Gatica, M. (2019). Modalidades represivas contra los trabajadores chilenos en la Patagonia argentina: el caso de los obreros expulsados de Chubut. Páginas, 12(28). Recuperado de: https://doi.org/10.3 5305/rp.v12i28.379

Scatizza, P. (2017). Autonomía y sistematicidad del dispositivo represor. La Policía Federal en Neuquén (1975-1978). Páginas, 9(21), 154-174. Recuperado de: https://doi.org/10.35305/rp.v9i21.276

Scocco, M. (2019). Las fuerzas represivas y las divisiones de inteligencia: El Ejército y las policías en Rosario (1966-1979). Revista de Estudios sobre Genocidio, (14), 11-28. Recuperado de: http://revistas.untref.edu.ar/ind ex.php/reg/article/view/329

Tapia, A.; Chávez, M. y Vezub, J. (2020). Tecnologías de identificación y clasificación social: el Fondo de Prontuarios Policiales del Chubut. Prohistoria, XXIII(34), 339-354.

\section{Notas}

1 Esto es, problematizando también las relaciones interpersonales al interior de la fuerza (vínculos jerárquicos, de funciones, posiciones, etc.); entre los diferentes organismos del Estado y de la sociedad política que configuran la "comunidad informativa"; y -al exterior-, la relación entre sus "espías" o agentes y los miembros de la sociedad civil en la que se infiltran y vigilan. A propósito de la división del trabajo y de las jerarquías establecidas, es sugerente la distinción entre información (datos y registros materiales) e inteligencia (análisis de esos datos y producción de ideas/planes), que planteaban los propios manuales militares (Funes, 2007). Esa distinción, no solo nos permite pensar las etapas del "circuito de inteligencia", sino que también nos señala que solo una "élite" (de carrera y con mayores conexiones políticas) tenía acceso al análisis integral de la información que recopilaban las agencias y de los datos que recababan sus "agentes de campo". Esa separación y fetichización de la producción de inteligencia, expresa también aspectos de la profunda relación entre saber y poder -que señalara Foucault.

2 Como sostiene Gabriela Aguila analizando el funcionamiento de la Dirección General de Investigaciones: “...si la vigilancia, espía y control de personas y organizaciones puede concebirse como una de las facetas del accionar represivo implementado por el Estado en esos años, la DGI formó parte activa de esa trama en tanto proveyó de información a quienes se ocuparon de perpetrar la persecución" (Aguila, 2013b, p. 23-24).

3 Si bien entendemos que la información de ese archivo excede lo estrictamente represivo, consignando otra gama de datos igualmente valiosos para el conocimiento histórico (como veremos en uno de los casos que abordamos), la razón de su confección no dejó de estar guiada por ese imperativo de lucha contra el "enemigo interno" o la "subversión", que es lo que le da especificidad histórica al archivo; la principal finalidad de la información allí consignada fue crear inteligencia y desplegar una burocracia que sirviese para la represión interna de la movilización social en ascenso. Por eso nos referimos a esa documental del APM como "archivo de la represión". A propósito del mismo explica Mónica Gatica que: "Aunque muchos actores locales sabíamos de su existencia, desde 1983 hasta Marzo de 2004 pensamos que había sido destruido. Resultó que circunstancialmente una historiadora, en el verano de 1998-1999, en medio de una remodelación de la antigua casa de gobierno encontró parte de este repositorio, y decidió 'esto hay que guardarlo, rescatarlo, porque era un documento histórico y con gran peso testimonial para la nueva etapa democrática', pero no denunció el hallazgo, y el mismo recién tomó estado público después de cinco años, cuando fue restituido. En ese período fue trasladado de modo bastante precario y conservado en domicilios particulares" (Gatica, 2010, p.166-167).

4 "Como hemos visto, la clandestinidad operativa era ineludible para el otro pilar del modelo francés: obtener la más completa información o 'hacer inteligencia' en la jerga angloamericana. Los encargados de conseguirla procedían de los Servicios y de la $2^{\circ}$ Jefatura, y además se formaron los Destacamentos Operativos de Protección (DOP). Tanta importancia adquirió la información 'que todos los oficiales se volvieron especialistas en ella', y ciertos conscriptos también" (Oliveira-Cézar, 2005, p. 125).

5 Manual RC 8-3, Operaciones contra fuerzas irregulares, 1969, p. 62.

6 Se trata de un plan del Ejército, que observa y evalúa la Armada Argentina (específicamente el SIN, Servicio Inteligencia Naval; este es el documento contenedor principal) a cargo de la Jefatura de Política y Estrategia de la Armada. Esta documentación fue incorporada al juicio por la Masacre de Trelew (2012) y su tratamiento forma parte del capítulo "Polo Represivo" de la tesis de doctorado de Axel Binder, en proceso de redacción. 
7 Armada Argentina, Jefatura de Política y Estrategia "Situación y Perspectivas de la "Comisión Coordinadora para la Acción de las Fuerzas Policiales de la Nación”, 1970.

8 Documento de la J.C.F. (LCS-106) “Pasaje a la ofensiva en la lucha contra la subversión”, 14/6/71; también se desprende de la documentación incorporada al juicio por la Masacre de Trelew (2012).

9 Ibíd, ARA, 1970.

10 La documentación correspondiente a la SIDE sobre “Averiguaciones de Antecedentes..." de personas que ingresan en la administración pública entre 1977-1979 (disponible en el Archivo Provincial de la Memoria Chubut), evidencia un alto grado de seguimiento y calificación de ciudadanos, en especial de origen chileno, lo que nos hace repensar la centralidad de los órganos de inteligencia, y el valor capital de esa información en tanto principal insumo represivo (a pesar de que, como ya dijimos, en el desarrollo de su actividad se consigne información más amplia que la del requerimiento inmediato ese: vigilar y castigar lo político/ideológico).

11 Militante de la Juventud Peronista de Trelew y uno de los dirigentes sociales del Trelewazo de 1972.

12 Enrique B. Himschoot, Jefe de Policía, 11 de febrero de 1969, APM. La Jefatura de Policía solicitaba antecedentes de 18 personas vinculadas a Teatro Estudio Trelew, de las que solo 6 se tenían datos identificatorios.

13 Se refiere a los Delitos contra la Lealtad a la Nación, del Código de Justicia Militar: "espionaje y revelación de secretos concernientes a la defensa nacional" e "infidelidad en el servicio"

14 "En 1970 un informe de inteligencia de la Prefectura Nacional Marítima de Comodoro Rivadavia (donde Bel trabajó como docente) considera como 'antecedentes desfavorables' de Elvio Ángel Bel que, en 1965 'concurría asiduamente al domicilio' de Macías y que, en 1963, 'fue miembro' del MEPES (Movimiento Estudiantil pro Enseñanza Superior)" (Fernández Picolo, 2014, p. 119).

15 Por ejemplo: "paralelamente se trató de determinar los medios de vida del causante, apareciendo entonces empleado en la librería propiedad del Señor Milton Arturo Belzunce, lugar donde se lo vió (sic) por varios días, desapareciendo posteriormente. Se encuentra vinculado a los integrantes del teatro independiente 'El Grillo' (...) se ha visto al causante en repetidas oportunidades acompañado de varios jóvenes desconocidos en la ciudad de Trelew” (Policía Federal, “Antecedentes", Bs.As. 21/02/1969).

16 Como que "Con fecha 23-02-1966 activa en el comunismo local y con otros de su misma ideología, busca acercamiento a peronistas, quienes se encuentran con graves disensiones internas (...) el informado figura en la localidad de Rawson, como infiltrado comunista" (Ibid, Policía Federal, 1969).

17 "Creemos que será posible constituir una filial patagónica de SADE, que actuará con especial referencia a los problemas nacionales. Escritores e intelectuales de la talla del Padre Entraigas, Feldman Josín y Porcel de Peralta, de la significación consular de Gregorio Álvarez, así como de muchos otros que se destacan en el campo cultural, permiten augurar una labor fecunda'. Siempre he creído - termina diciendo Ricardo Mosquera- que la Patagonia es uno de los grandes laboratorios del destino nacional, la nueva frontera argentina" (Diario Jornada, 12/06/1970, p. 8).

18 SI-Chubut, 26/06/1970, APM.

19 En el pedido dirigido a la SIDE faltaban datos filiatorios de algunos de los participantes del encuentro, ante lo que el SI Chubut se disculpaba por "apartarse de las disposiciones vigentes sobre aporte de datos de identidad y filiación de las personas de quienes se solicitan antecedentes, en razón de carecerse de los mismos” (Ibid.). El pedido realizado a la Subsecretaría de Educación, meses más tarde, pudo haber tenido la finalidad de conseguir datos más precisos para precisar la búsqueda de los antecedentes políticos/ideológicos solicitados.

209 de Chubut y 2 de La Pampa; sobre una de las personas de Buenos Aires se solicitan mayores datos por haber varias personas bajo el mismo nombre; lo mismo uno de los escritores de Neuquén y otro del que se esperaban aún datos de la Policía Federal

21 Comisión Argentina de Calificación Ideológica Extremista, creada en 1967 a través de la ley 17.401 e integrada por agentes de inteligencia. Dependiente de la SIDE, se encargaba de calificar publicaciones y personas como "comunistas". Es interesante la observación de Funes (2008) destacando la "inflación semántica” de los "comunistas" que ahora pasan a ser catalogados como "extremistas". La intervención de la CACIE "producía como efecto un tipo de 'muerte civil' que castigaba a las personas 'inhabilitándolas' para el usufructo de una variedad de derechos civiles y sociales. La liberación de un detenido a disposición del poder ejecutivo nacional también era decidida por la 'Comunidad Informativa' que integraban los titulares de los distintos organismos de inteligencia" (Fernández Picolo, 2014, p. 107).

22 SI-Chubut, 26/06/1970, APM.

23 Jefatura Policía de Chubut, “Asunto: informar antecedentes”, 2/07/1970, APM.

24 SI-Chubut, "Informe Primer Encuentro Escritores", en Legajo nº 15 de Actividades Culturales, APM Chubut, 1970

25 Ibid, SI-Chubut, "Informe Primer Encuentro Escritores".

26 No obstante, la ausencia de membretes, fechas o sellos (sumado a la dificultad de que los documentos de esta sección no guardan una clara secuencia cronológica) no nos permiten corroborar que haya sido la Jefatura de Policía el organismo productor. Además, el informe contiene datos sobre escritores (en especial de otras provincias), que no figuran en el 
pedido original que el SI-Chubut realiza a la Policía; y que sí lo hacen en el requerimiento a la SIDE. Sin embargo, el orden de las personas informadas de Chubut, es tal cual figura en el requerimiento a la policía.

27 SI Chubut, "Solicitar información...”, 15/02/1972 APM.

28 El número 8/9 de abril/mayo de 1971 fue dedicado al encuentro de Trelew bajo el título "Trelew: muestra y propuesta". Allí se consignó que: "El esfuerzo organizativo (encabezado por Rudy Miele) enfrentando la presión exterior (los grupos que iban llegando") hizo estallar la santabárbara. De pronto Trelew dejaba de ser una plaza pequeña y provinciana, tremendamente acomplejada, y se ponía en marcha. Se producen abandonos y radicalizaciones, planes futuros y entierros (...) se buceó a tanta profundidad que la realización de Trelew II peligra (...) Tanta y tan ruidosa fue la ruptura. Por primera vez un festival sin bombos y platillos componía una música de circo. Algo muy importante se había descubierto: el festival se había desnaturalizado. Ya no lo era. Un seminario aparecía en su lugar. Y Seminario a varios niveles: artístico, ideológico, político, económico y técnico / No todos los grupos lo entendieron así, por supuesto. Pero sí la mayoría / En Trelew nació una posibilidad de organización revolucionaria" ("Trelew: muestra y propuesta", Teatro 70 n8-9 AbrilMayo, cedebaires, 1971, p. 18. Subrayado nuestro).

29 Hasta informan que el alojamiento de los elencos y directores "se organizó en una casa de familia perteneciente a un integrante del Teatro Estudio Trelew, desocupada al efecto. Las camas, frazadas, etc. fueron cedidas por la Base Aeronaval de Trelew" (Ministerio de Gobierno Educación y Justicia, “Memorándum”, 2/03/1971); y hasta les fue facilitado uno de sus colectivos para que recorriesen la zona. Esta colaboración estrecha de la Armada podría tener que ver también con su especial interés en las actividades políticas y culturales, vigilando "de cerca" posibles contenidos "izquierdistas" o "extremistas”; o bien con su política de relaciones públicas y su capacidad para insertarse en la sociedad civil.

30 Ministerio de Gobierno Educación y Justicia, “Memorándum”, 2/03/1971.

31 Ibid, Ministerio de Gobierno, Educación y Justicia, 1971.

32 Parte SIDE No01531/183, 1973, APM.

33 SI-Chubut, "Espectáculos de contenido ideológico", 14/02/1973, APM.

34 No hay respuesta asociada a ese documento por lo que queda "suelto", fuera de la sección o subserie: Se requería "1) nómina de integrantes (...), 2) antecedentes judiciales, contravencionales y vecinales que registraron, 3) antecedentes políticos e ideológicos que registrasen, 4) apellidos y nombres de sus empresarios (...) 5) obras teatrales realizadas y por quién fueron dirigidas, 6) "giras efectuadas por el elenco teatral, 7) todo dato o circunstancia que se estime de interés consignar” (Jefatura de Policía de Chubut, nota nro. 22 D.I.P., “objeto: recabar información”, 25/02/1972).

35 SI-Chubut, "Espectáculos de contenido ideológico”, 30/04/1973, APM. Subrayado en el original.

36 SI-Chubut, 30/04/1973, APM.

37 SIDE, “parte 3383",05/04/1973, APM.

38 SIDE, "Directivas para el trato de documentación”, anexo al parte 3383.

39 Funes (2007) señala que especialmente luego del Cordobazo, se comienza a generalizar la categoría de "delincuente subversivo", pasando de "izquierdista" a "extremista”. Es interesante como Funes observa en el caso del Centro Editor de América Latina, que los servicios de inteligencia hacia 1969 lo clasificaban bajo el rótulo de "comunismo", y ya para 1980, el mismo material era catalogado en la sección de "delincuente subversivo". Es muy importante esto porque muestra que lo que va cambiando es la mirada que se tiene desde el poder; los libros siguen siendo los mismos, lo que cambia es la "valoración" de su peligrosidad (en función de su capacidad de ruptura o poder de crítica al statu-quo y al sistema de dominación).

40 Lo mismo observa Gabriela Aguila respecto de la Policía de Santa Fe, sosteniendo que "en ese contexto crecientemente represivo, las policías adecuaron su estructura y funcionamiento a perseguir a la "subversión", utilizando métodos ya probados en la lucha contra la delincuencia común y/o incorporando prácticas y dispositivos represivos más o menos novedosos, lo que se amplificó cuando las FFAA asumieron el comando de la "lucha antisubversiva" y pusieron bajo su control operativo a la policía y las fuerzas de seguridad” (Aguila, 2018b, p. 130).

41 Falta aún acceder a la normativa policial para ver reglamento del DIP en Chubut, pero suponemos que no debería apartarse demasiado del de otras provincias. Sobre el Servicio de Informaciones de la Jefatura policial de Rosario, analizando su reglamento Gabriela Águila consigna que su misión era "reunir, procesar y difundir toda información que permita conocer la real situación de las organizaciones delictivas que operan en el ámbito de su provincia”, vinculado con 'delitos comunes, actividades extremistas y todo lo que haga a los asuntos políticos, gremiales, estudiantiles, migratorios, turísticos, económicos, etcétera, que tengan incidencia en el ámbito provincial'. Asimismo, entre sus misiones y funciones estaba cooperar con otros departamentos de informaciones policiales y organismos de inteligencia” (Águila, $2018 \mathrm{~b}, \mathrm{p}$. 135). Por su parte, Celeste Schnyder y Paulo Margaria, analizando el DIP de la provincia de Santiago del Estero (cuya inicio de actividades sitúan en 1972) explican que el organismo tenía la "función de reunir información suponía el registro, clasificación, archivo de las noticias publicadas, datos surgidos de seguimientos y escuchas telefónicas, informes ambientales sobre las actividades, públicas y privadas, de ciudadanos 'marcados', en razón de su pensamiento político y militancia como blancos de espionaje” (Margaria y Schnyder, 2013, p. 6). 
42 La directiva No2/71 "Pasaje a la ofensiva en la lucha contra la subversión", en su punto 3.8.3 convenía que la Armada y la Fuerza Aérea operarían en sus respectivas jurisdicciones establecidas en el Plan de Contención Marco Interno 70/71. La jurisdicción de la Armada, la que nos interesa para nuestra región, era la considerada "natural" de la fuerza: el mar, los ríos navegables, sus riberas, zonas portuarias y la zona territorial circundante a las bases y unidades de tierra, manteniendo además el control operacional de la Policía territorial de Tierra del Fuego. Nuestra hipótesis es que en este momento, la región Noreste de Chubut pasa a quedar bajo el influjo de la estrategia represiva de la Armada: el Plan de Contención Interna de la Armada Argentina (PLACINTARA). PLACINTARA 72 estableció 11 Fuerzas de Tareas (FUERTAR) distribuidas territorialmente. Nuestra región, cayó bajo la jurisdicción de la FUERTAR 7, denominada "Agrupación Trelew”, al mando del Jefe de la Base Aeronaval A. Zar, con dominio sobre los establecimientos y dependencias de la Armada y Prefectura en Trelew, Rawson, Golfo Nuevo, Península Valdéz y las zonas portuarias de Madryn y Rawson así como también los Departamentos de Telsen, Gastre, Gaiman. Las unidades a su disposición eran el Batallón Infantería de Marina 4 (BIM 4), el Apostadero Naval Golfo Nuevo, la Prefectura Madryn y Rawson. Así mismo, se le sumaban las secciones de inteligencia de la Prefectura de Comodoro, Pto. Madryn y Rawson. 